\title{
Consistent contrast between eyelid and iris brightness supports a role for vigilance signalling in ducks
}

\author{
MATTHIEU GUILLEMAIN, ${ }^{1 *}$ CAROL FOUQUE ${ }^{2} \&$ JORDI FIGUEROLA ${ }^{3}$ \\ ${ }^{1}$ Office National de la Chasse et de la Faune Sauvage, CNERA Avifaune Migratrice, La Tour du Valat, Le Sambuc, \\ F-13200, Arles, France \\ ${ }^{2}$ Office National de la Chasse et de la Faune Sauvage, CNERA Avifaune Migratrice, Station de la Dombes, Montfort, \\ F-01130, Birieux, France \\ ${ }^{3}$ Estación Biológica de Doñana, CSIC, c/Americo Vespucio s/n, 41092, Isla de la Cartuja, Seville, Spain
}

\begin{abstract}
Signalling self-ability to maintain vigilance may help in securing a mate, while providing accurate information about vigilance status may result in conspecifics adjusting their own scanning rate of the environment, potentially to the individual's benefit. In birds, vigilance is often associated with head-up postures adopted within a bout of head-down activity, and this can be used by conspecifics to assess the vigilance of their flock mates. However, vigilance behaviour is not always obvious and other cues may then be used to assess vigilance rates of conspecifics. Here we assess whether iris/eyelid/face patterns from 43 duck species are consistent with the hypothesis that eyelid brightness has evolved so as to contrast with iris brightness, which may then help in signalling individual vigilance status. Ducks generally flock when resting during the day, and because of their wide visual fields, individuals can monitor their environment while remaining in a resting head-down position. Ducks also show a wide variety of plumage and iris patterns, with both light-headed and dark-headed species. Matching our prediction, most ducks with dark irises had pale eyelids, irrespective of head colour. Furthermore, the smaller number of species with a pale iris generally have darker eyelids. A phylogenetic analysis shows a clear and significant association in the evolution of eyelid and iris brightness patterns in both males and females. These data therefore provide support for the hypothesis that eyelid brightness has evolved to act as a contrast with iris brightness. Further studies are now needed to examine the extent to which and the way this is used in vigilance information transfer between individuals.
\end{abstract}

Keywords: Anatidae, evolution, resting ducks, vigilance.

Being able to accurately inform others about selfvigilance status is a crucial requirement. First, advertising the ability to maintain high vigilance rates may help secure a mate (e.g. Dahlgren 1990). Secondly, while it had long been thought that members of a group scan the environment independently of each other (e.g. Pulliam 1973), it is now increasingly clear that individuals adjust their vigilance to that of their neighbours (Pays

*Corresponding author.

Email: matthieu.guillemain@oncfs.gouv.fr et al. 2010), either through co-ordination (by being vigilant when neighbours are not, thereby avoiding overlapping vigilance bouts; e.g. Rodriguez-Gironés \& Vásquez 2002) or through synchronization of vigilance (by increasing vigilance rate when neighbours are more vigilant; e.g. Pays et al. 2007a, Sirot \& Touzalin 2009). The individual benefit of signalling vigilance status is obvious when there is co-ordination: indicating non-vigilant status should lead to conspecifics increasing their own vigilance, thereby improving an individual's safety. However, in flocks where vigilance is 
synchronized, the proportion of time an individual spends on vigilance has also been shown to decrease with increasing group size (Pays et al. 2007a). In such a situation, it should benefit individuals to advertise their vigilance status to let neighbours synchronize their own behaviour.

Scanning the environment for predators or competitors is often associated with overt head-up postures, making it easy for individuals to monitor the vigilance status of each other (e.g. Bertram 1980). However, animals also maintain some level of vigilance even when the head is lowered (a less conspicuous form of vigilance behaviour; Lima \& Bednekoff 1999, Guillemain et al. 2001). In such cases, flock mates may rely on more subtle cues to assess whether their neighbours are vigilant, and adjust their own behaviour accordingly. We hypothesize that bird eyelids may in some species have evolved such that eyelid colour and iris brightness would contrast, and could then be used as a signal by which individuals in resting position can advertise their vigilance level: a clear signal that they have their eyes open (vigilant) or closed (sleeping).

Wintering ducks (Anatidae) typically rest in flocks during the day (Tamisier 1978, Guillemain et al. 2010). The ability to detect approaching predators (mostly predatory birds) is a major driver of such diurnal gregarious behaviour (Tamisier 1985). Ducks rely on an upright head position to scan their environment but are also able to maintain vigilance while resting. In this situation, the head is lowered with the bill tucked in among the back feathers, but vigilance usually relies on regular opening of the eyes (e.g. Gauthier-Clerc et al. 1998, Rattenborg et al. 1999). This is made possible by the wide visual fields of ducks, which provide complete or almost complete coverage of the hemisphere around and above the head (Martin 1986, Guillemain et al. 2002).

It should be necessary for flock-resting ducks to advertise their vigilance status to each other, and because the different duck species differ in iris and head plumage colour patterns, ducks are particularly appropriate species with which to test the above hypothesis about the evolution of contrast between eyelid colour and iris brightness. Most duck species have dark irises (see illustrations in del Hoyo et al. 1992). We therefore predicted that most ducks would have pale eyelids, irrespective of general head plumage colour or pattern. Thus, if an eye is closed while the bird is sleeping, this would be signalled by the clearly visible pale eyelid. Conversely, when the bird is in a resting posture but is being vigilant, i.e. at least one eye is open, this eye would appear dark. Although less frequent, in some duck species differences in iris colour (either whitish or yellow) among age or sex classes provides a further opportunity to test this hypothesis: ducks with a pale eye should have darker eyelids, irrespective of head colour or plumage pattern. In this case, vigilance status would be signalled by the reverse signal, with open eyes appearing light and closed eyes appearing dark.

\section{METHODS}

We examined 43 species of duck housed in the collection of the Parc des Oiseaux, a zoological park mostly dedicated to the exhibition of bird species from around the world, located in Villars-les-Dombes, eastern France $\left(46^{\circ} 0^{\prime} 09^{\prime \prime} \mathrm{N}\right.$, $\left.05^{\circ} 01^{\prime} 47^{\prime \prime} \mathrm{E}\right)$ on 12 June 2011. We examined live ducks rather than museum specimens because eyelids are not always visible on stuffed birds, especially mounted ones. The sample represented 71 different plumage morphs (11 sexually monomorphic and 32 sexually dimorphic species, not all morphs of dimorphic species being on display; see Supporting Information Table S1). We chose the observation date such that all male ducks would be in breeding plumage (male Baikal Teals Anas formosa were in cryptic eclipse plumage and were therefore omitted from our dataset). We observed birds at a distance of 1-15 m, using 10942 binoculars when necessary. Each morph was assigned to one of two irises, and one of three eyelid brightness categories. Irises were classified as pale or dark, and an additional dull category was used for grey or light brown eyelids of intermediate brightness. Classification was conducted separately by two observers (C.F., M.G.) and was unambiguous, as both observers always obtained the same results. We also scored the general brightness of head plumage around the eye as pale or dark (Table S1). The aim was not to determine brightness quantitatively, which would require the use of a spectrometer parameterized with duck visual system characteristics (e.g. Legagneux et al. 2010), but only to assess whether a simple brightness score of two (iris and head) or three (eyelid) categories would provide support for the hypothesis of contrasting iris/eyelid brightness 
evolution described above. Only one or a few bird pairs were on display for most species, although many individuals could be observed for very common taxa such as Mallard Anas platyrhynchos. In the latter case, no inter-individual differences in eyelid brightness category assignment were noted. We examined eyelids when ducks were either resting or preening. It is the external eyelid, i.e. the mobile and feathered part of the skin covering the eye when closed (mostly the lower eyelid, which is more mobile), that we consider here, and not the internal nictitating membrane, or third eyelid, which is typically transparent or slightly opaque. Although captivity may affect bird coloration and brightness (e.g. owing to artificial diet), we did not detect any difference in eyelid and iris brightness category between captive birds and wild ones in the field among the seven European species examined (M. Guillemain unpubl. data).

Whether dark irises were more frequently associated with pale eyelids than random, whether the opposite result held for pale irises, and whether a clear pattern of contrast between iris brightness (dark and pale irises pooled) and eyelid brightness existed were tested using chi-squared tests. Because dull eyelids contrasted on both dark and pale irises, these were considered dark for paleeyed birds and pale for dark-eyed birds.

A phylogenetic analysis was then used to assess whether evolutionary changes in iris brightness were associated with changes in eyelid brightness category. The phylogeny was derived from the working phylogeny assembled in Figuerola and Green (2000). The trees were constructed using MACCLADE 4.06 (Maddison \& Maddison 2003) and character evolution reconstructed using parsimony algorithms. To analyse the relationship between changes in eyelid and changes in iris brightness category we used the program DISCRETE (Pagel 1994). The polytomies in the phylogeny were resolved parsimoniously in relation to character changes. Association between eyelid and iris colour was assessed by likelihood ratio tests comparing models of dependent and independent trait evolution, and significance was estimated by comparison with 100 simulations (Pagel 1994). Eyelid colour was coded in two different ways: (1) dark (including dark and dull) versus pale; or (2) dark versus pale (including dull and pale). Analyses were conducted separately for males and females.

\section{RESULT S}

The majority (61 of 71) of duck morphs examined had a dark iris. Most $(n=51)$ had a pale eyelid, and 60 had dark head plumage. A dark iris was associated with a paler eyelid (Pale or Dull categories) far more frequently than expected by random $\left(\mathrm{V}^{2}=53.26, \quad \mathrm{df}=1, \quad \mathrm{P}<0.0001 ; \quad\right.$ Table 1$)$. The only exceptions were the monomorphic Philippine Duck Anas luzonica and male Australian Shelduck Tadorna tadornoides, both of which had a dark iris and dark eyelid. Apart from a few cases of pale eye-rings (see Table S1) and species such as the Plumed Whistling Duck Dendrocygna eytoni and Ruddy Shelduck Tadorna ferruginea, which had light head plumage, ducks with a dark iris had a dark head in addition to pale eyelids. Eyelid colour therefore always contrasted strongly with head plumage colour when closed (e.g. resting Eurasian Teal Anas crecca in Fig. 1).

Only 10 morphs from seven duck species had a pale iris. Among these, only one species (Rajah Shelduck Tadorna radjah) had pale eyelids. Five pale-eyed morphs had dull eyelids, i.e. not as bright as in the species above, but these differed enough from a pale iris to allow immediate assessment of eye-opening status (Table 1). Furthermore, female Tufted Duck Aythya fuligula and both sexes of the Goldeneye Bucephala clangula, which had pale irises, had almost black eyelids. The most striking example in this category was apparent in the Ferruginous Duck Aythya nyroca, which is almost sexually monomorphic, the sexes differing only in the brightness of the iris: both sexes have a relatively similar brownish head plumage (although females are slightly duller than males) but males have a whitish iris, whereas females have a dark brown iris. Following the same pattern as above, female Ferruginous Ducks had a pale eyelid, whereas the male had a

Table 1. Number of duck morphs in each combination of iris and eyelid brightness categories. Each morph was assigned to one of two classes of iris brightness and one of three classes of eyelid brightness.

\begin{tabular}{llcc}
\hline & \multicolumn{3}{c}{ Eyelid brightness } \\
\cline { 2 - 4 } Iris brightness & Dark & Dull & Pale \\
\hline Dark & 2 & 9 & 50 \\
Pale & 4 & 5 & 1 \\
\hline
\end{tabular}



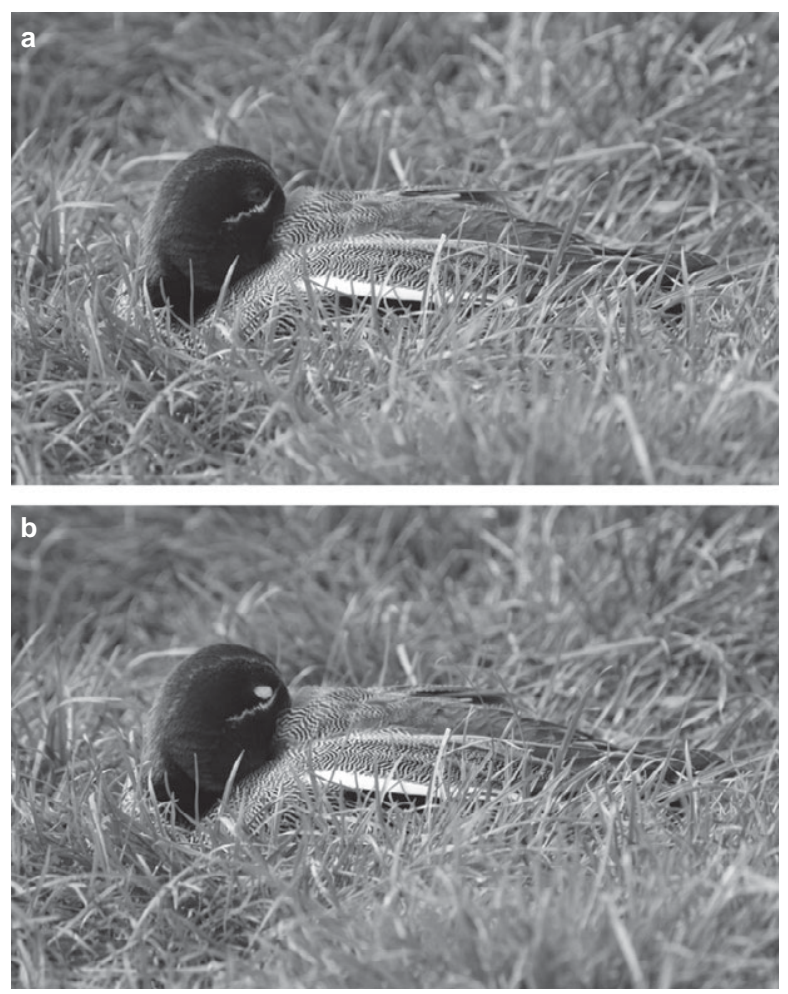

Figure 1. The same resting male Eurasian Teal while being vigilant (a) and sleeping (b).

brown eyelid. The likelihood of having darker (i.e. Dark or Dull) eyelids in pale-eyed birds differed significantly from random $\left(\mathrm{V}^{2}=6.40, \quad \mathrm{df}=1\right.$, $\mathrm{P}=0.0114$; Table 1).

When both dark-eyed and pale-eyed species were considered together, 68 of 71 morphs had contrasting iris and eyelid brightness scores $\left(\mathrm{V}^{2}=57.93\right.$, df $=1, \mathrm{P}<0.0001$; Table 1).

The phylogenetic analyses demonstrated a clear association in the evolution of iris brightness and eyelid brightness characters, regardless of how dull eyelids were considered, both in males (Dull considered Dark: $\mathrm{P}=0.04$; Dull considered Pale: $\mathrm{P}<0.01$; Fig. 2) and in females (Dull considered Dark: $\mathrm{P}<0.01$; Dull considered Pale: $\mathrm{P}=0.02$; Fig. 3).

\section{DISCUSSION}

The duck species examined supported our prediction that eyelid and iris coloration exhibits contrasting brightness, irrespective of both head and iris colour. In the limited number of diving duck species observed with pale irises, the dark eyelids were often similar to the rest of the head plumage (the same pattern also occurs in Lesser Scaup Aythya affinis, A. Afton pers. comm.). Conversely, most ducks have both dark eyes and dark head plumage, such that differentiated pale eyelids may have evolved under specific constraints. The contrast between pale eyelids and the rest of the head is reinforced by the presence of dark eye stripes in many of these species. Eye structure and vision processes are well known in birds (e.g. Martin 1985, Zeigler \& Bishof 1993), yet virtually no study has investigated eyelid coloration and brightness apart from authors making use of eyelid colour as an aid to species identification (e.g. Larkin 2000 to discriminate American Anas americana from Eurasian Anas penelope Wigeon in the field). Note that the term eyelid (or eye-lid) has also been used to describe eye-rings in some birds, as well as the coloration of the bare patch of skin surrounding the eye in some species of birds (e.g. Greig-Smith 1984). This, however, differs from the use of the term eyelid as defined here (see Methods).

Our data suggest that natural selection has promoted the development of a strong contrast between eyelid and iris brightness, regardless of head plumage or iris coloration, which is supported by the phylogenetic analyses. Eyelid colour has not been reported to play a role in vision or any physiological process (G.R. Martin pers. comm.). In contrast to iris colour and brightness, which may be involved in mate choice (Newton \& Marquiss 1982, Massaro et al. 2003, see Craig \& Hulley 2004 for a more general assessment of bright eye evolution in passerines), eyelids are generally open when the bird is active, such that eyelids are unlikely to play a role in sexual displays. Given that eyelid colour is visible only when the eye is closed, we consider the strong contrast between brightness of the eyelid and iris to have been selected upon to advertise eye closure. This would match Danchin et al.'s (2007) definition of a signal: 'in signalling, individuals expect a benefit from the receptors' reaction to the information carried by the signal'.

It is increasingly recognized that social animals do not scan their environment independently from each other but rather adjust their vigilance to that of their neighbours (Beauchamp 2009, Sirot \& Touzalin 2009, Pays et al. 2010). If vigilance is mostly co-ordinated in flocks of ducks, i.e. birds tend to be more vigilant when their neighbours are not, and vice versa (e.g. Rodriguez-Gironés \& Vásquez 2002), eyelid brightness should 

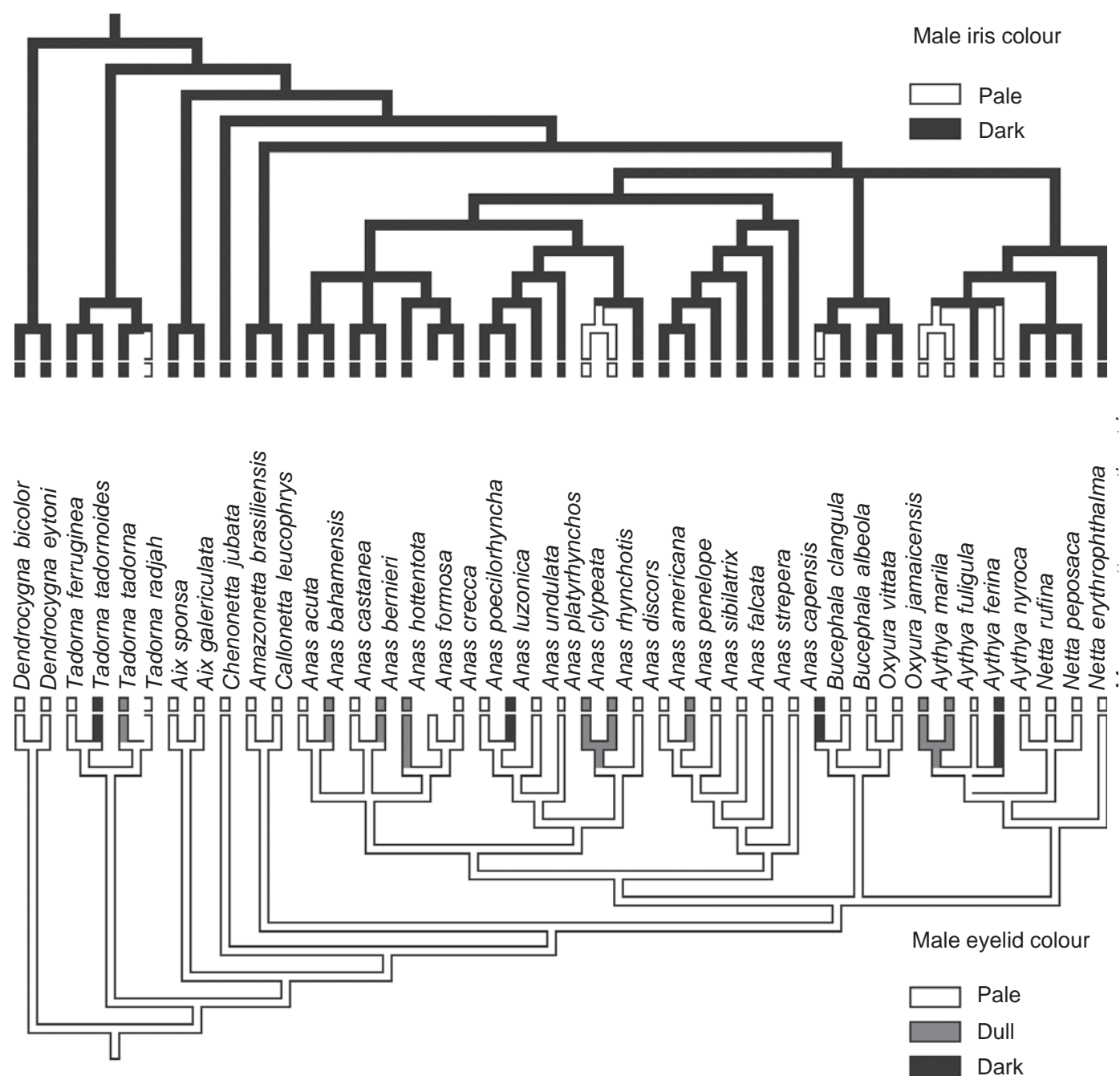

Figure 2. Evolution of iris and eyelid brightness in male ducks traced on the phylogeny presented in Figuerola and Green (2000). The states of interior nodes were optimized using parsimony in MACCLADE (Maddison \& Maddison 2003).

theoretically help transmit an honest signal, as cheaters (individuals potentially able to hide the fact that their eye is closed) would actually decrease their own safety by not eliciting an increased vigilance rate in their neighbours. Because the apparent strong contrast between iris and eyelid brightness makes the information about eye-opening status more obvious, and hence less costly for neighbours to acquire, the observed coloration pattern may actually promote co-ordinated rather than synchronized vigilance (RodriguezGironés \& Vásquez 2002, Sirot \& Touzalin 2009).

Conversely, it is also possible that ducks synchronize their vigilance, i.e. an individual becomes more vigilant when its neighbours are already vigilant, potentially leading to waves of vigilance within groups (e.g. Pays et al. 2007a). Although some birds have been reported to show waves of vigilance without having contrasting iris/eyelid brightness (e.g. gulls, Beauchamp 2011), any means of enhancing vigilance signalling should promote vigilance synchronization in such systems. Synchronization of vigilance may be beneficial to each individual through group cohesion (reviewed by Pays et al. 2007b), but individual vigilance has also been reported to decrease with increasing group size even when vigilance is synchronized (Pays et al. 2007a). Contrasting iris/eyelid brightness may therefore always bring a benefit and hence have evolved in ducks independently from the way conspecifics use the signal, i.e. either for co-ordination or synchronization of their own vigilance. 

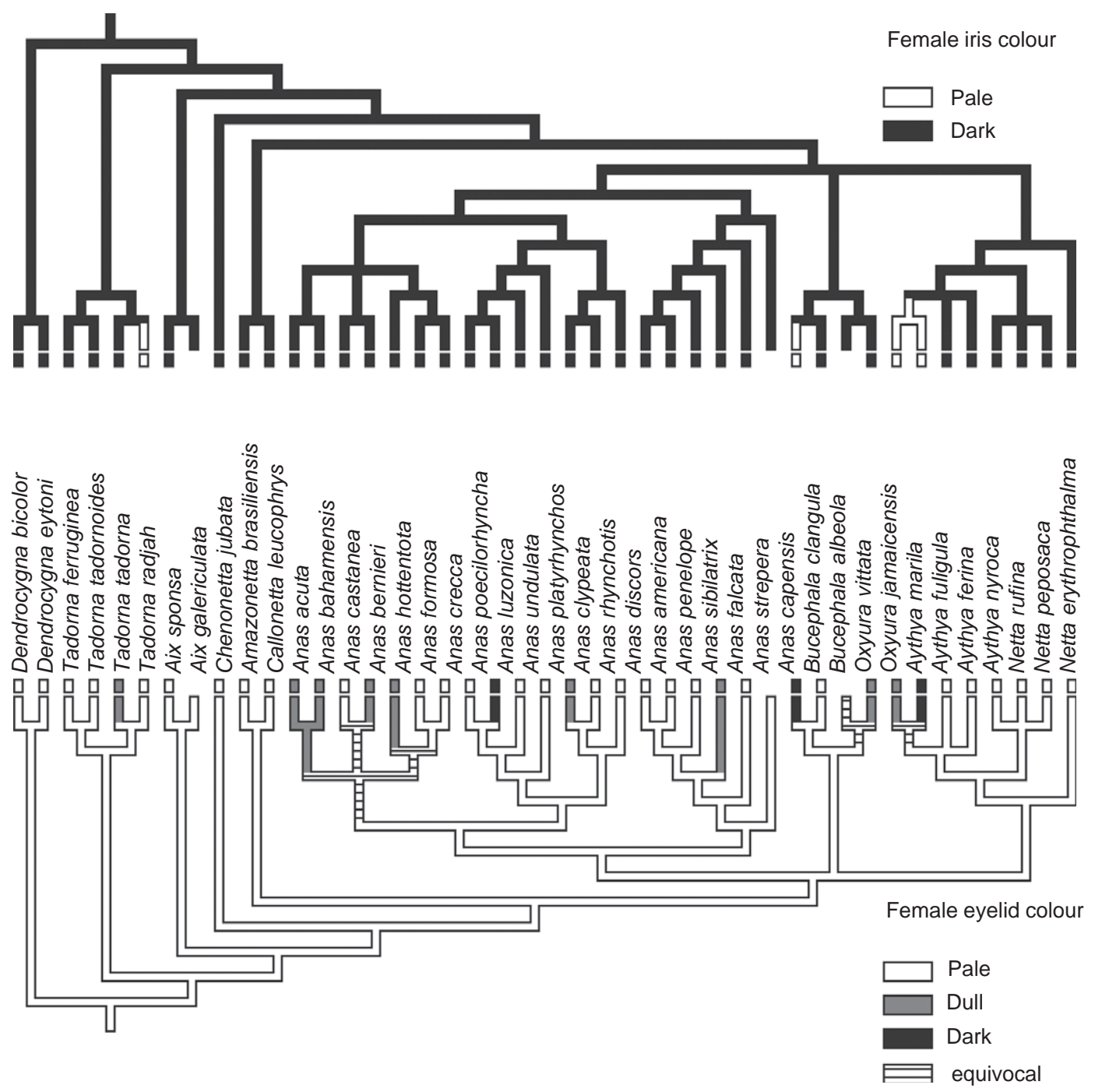

Figure 3. Evolution of iris and eyelid brightness in female ducks traced on the phylogeny presented in Figuerola and Green (2000). The states of interior nodes were optimized using parsimony in MACCLADE (Maddison \& Maddison 2003).

To test the hypothesis that eyelid brightness is used as a signal, future studies should aim to demonstrate that conspecifics do use such information in a way that is beneficial to the individual displaying its eyelids. This could be documented through measurements of flock mate vigilance depending on an individual's eye-opening frequency, or through experimental manipulation of eyelid brightness in live or plastic decoy ducks, and then observing the resulting vigilance of neighbouring individuals. However, flocking ducks probably do not distribute alertness randomly between the two cerebral hemispheres during sleep; for instance, individuals may favour opening of the eye on the outer side of the flock
(Rattenborg et al. 1999). Additionally, birds show some laterality in vigilance, favouring one eye over the other (e.g. Franklin \& Lima 2001). Demonstrating that conspecifics are able to use individual eye-opening rate to adjust their own vigilance, and assessing the way they adjust their vigilance (i.e. co-ordination versus synchronization), is likely to prove challenging.

We would like to thank G. R. Martin, C. Eraud, B. Clark and A. Afton for useful advice, as well as E. Bureau and L. Simon for their hospitality at the Parc des Oiseaux. We thank K. Tarvin, R. Bowie and two anonymous referees for valuable comments on an earlier version of the manuscript. 


\section{REFERENCES}

Beauchamp, G. 2009. Sleeping gulls monitor the vigilance behaviour of their neighbours. Biol. Lett. 5: 9-11.

Beauchamp, G. 2011. Collective waves of sleep in Gulls (Larus spp.). Ethology 17: 326-331.

Bertram, B.C.R. 1980. Vigilance and group size in ostriches. Anim. Behav. 28: 278-286.

Craig, A.J.F.K. \& Hulley, P.E. 2004. Iris colour in passerine birds: why be bright-eyed? S. Afr. J. Sci. 100: 584-588.

Dahlgren, J. 1990. Females choose vigilant males: an experiment with the monogamous grey partridge, Perdix perdix. Anim. Behav. 39: 646-651.

Danchin, E., Giraldeau, A.L. \& Wagner, R.H. 2007. An information-driven approach to behaviour. In: Danchin, E., Giraldeau, L.A. \& Cézilly, F. (eds) Behavioural Ecology: an Evolutionary Perspective on Behaviour: 97-130. Oxford: Oxford University Press.

Del Hoyo, J., Elliott, A. \& Sargatal, J. (eds) 1992. Handbook of the Birds of the World, Vol. 1. Barcelona: Lynx Edicions.

Figuerola, J. \& Green, A.J. 2000. The evolution of sexual dimorphism in relation to mating patterns, cavity nesting, insularity and sympatry in the Anatidae. Funct. Ecol. 14: 701 $-710$.

Franklin, W.E. III \& Lima, S.L. 2001. Laterality in avian vigilance: do sparrows have a favourite eye? Anim. Behav. 62: 879-885.

Gauthier-Clerc, M., Tamisier, A. \& Cézilly, F. 1998. Sleepvigilance trade-off in Green-winged Teals (Anas crecca crecca). Can. J. Zool. 76: 2214-2218.

Greig-Smith, P.W. 1984. Changes in the eye-lid colour of Long-tailed Tits Aegithalos caudatus. Bird Study 31: 35-38.

Guillemain, M., Duncan, P. \& Fritz, H. 2001. Switching to a feeding method that obstructs vision increases head-up vigilance in dabbling ducks. J. Avian Biol. 32: 345-350.

Guillemain, M., Martin, G.R. \& Fritz, H. 2002. Feeding methods, visual fields and vigilance in dabbling ducks (Anatidae). Funct. Ecol. 16: 522-529.

Guillemain, M., Devineau, O., Brochet, A.L., Fuster, J., Fritz, H., Green, A.J. \& Gauthier-Clerc, M. 2010. What is the spatial unit for a wintering Teal Anas crecca? Weekly day-roost fidelity inferred from nasal saddles in the Camargue, Southern France. Wildl. Biol. 16: 215-220.

Larkin, P. 2000. Eyelid colour of American Wigeon. Br. Birds 93: 39-40.

Legagneux, P., Théry, M., Guillemain, M., Gomez, D. \& Bretagnolle, V. 2010. Condition dependence of iridescent wing flash-marks in two species of dabbling ducks. Behav. Processes 83: 324-330.

Lima, S.L. \& Bednekoff, P.A. 1999. Back to the basics of antipredatory vigilance: can nonvigilant animals detect attack? Anim. Behav. 58: 537-543.

Maddison, D. \& Maddison, W.. 2003. MacClade, Version 4.06. Sunderland, MA: Sinauer Associates

Martin, G.R.. 1985. Eye. In King, A.S. \& McLelland, J. (eds) Form and Function in Birds, Vol. 3: 311-373. New York: Academic Press.

Martin, G.R. 1986. Total panoramic vision in the mallard duck, Anas platyrhynchos. Vision Res. 26: 1303-1306.

Massaro, M., Davis, L.S. \& Darby, J.T. 2003. Carotenoidderived ornaments reflect parental quality in male and female Yellow-eyed Penguins (Megadyptes antipodes). Behav. Ecol. Sociobiol. 55: 169-175.

Newton, I. \& Marquiss, M. 1982. Eye colour, age and breeding performance in Sparrowhawks Accipiter nisus. Bird Study 29: 195-200.

Pagel, M. 1994. Detecting correlated evolution on phylogenies: a general method for the comparative analysis of discrete characters. Proc. R. Soc. B 255: 37-45.

Pays, O., Renaud, P.C., Loisel, P., Petit, M., Gerard, J.F. \& Jarman, P.J. 2007a. Prey synchronize their vigilant behaviour with other group members. Proc. R. Soc. B 274: 1287-1291.

Pays, O., Jarman, P.J., Loisel, P. \& Gerard, J.F. 2007b. Coordination, independence or synchronization of individual vigilance in the eastern grey kangaroo? Anim. Behav. 73: 595-604.

Pays, O., Blomberg, S.P., Renaud, P.C., Favreau, F.R. \& Jarman, P.J. 2010. How unpredictable is the individual scanning process in socially foraging mammals? Behav. Ecol. Sociobiol. 64: 443-454.

Pulliam, H.R. 1973. On the advantages of flocking. J. Theor. Biol. 38: 419-422.

Rattenborg, N.C., Lima, S.L. \& Amlaner, C.J. 1999. Halfawake to the risk of predation. Nature 397: 397-398.

Rodriguez-Gironés, M.A. \& Vásquez, R.A. 2002. Evolutionary stability of vigilance coordination among social foragers. Proc. R. Soc. B 269: 1803-1810.

Sirot, E. \& Touzalin, F. 2009. Coordination and synchronization of vigilance in groups of prey: the role of collective detection and predator's preference for stragglers. Am. Nat. 173: 47-59.

Tamisier, A. 1978. The functional units of wintering ducks: a spatial integration of their comfort and feeding requirements. Verh. Orn. Ges. Bayern 23: 229-238.

Tamisier, A. 1985. Some considerations on the social requirements of ducks in winter. Wildfowl 36: 104-108.

Zeigler, H.P. \& Bishof, H.J. 1993. Vision, Brain, and Behavior in Birds. Cambridge, MA: A Bradford Book/The MIT Press.

Received 23 August 2011; revision accepted 29 April 2012. Associate Editor: Keith Tarvin.

\section{SUPPORTING INFORMATION}

Additional Supporting Information may be found in the online version of this article:

Table S1. Iris, eyelid and head plumage brightness pattern in each of the 71 duck morphs examined.

Please note: Wiley-Blackwell are not responsible for the content or functionality of any supporting materials supplied by the authors. Any queries (other than missing material) should be directed to the corresponding author for the article. 